\title{
Analysis and Design of a High-Current, High-Voltage Accurate Power Supply for the APS Storage Ring*
}

\author{
M. Fathizadeh \\ Argonne National Laboratory \\ 9700 South Cass Avenue, Argonne, IL 60439
}

\section{Abstract}

There are 81 dipole magnets contained in the storage ring at the Advanced Photon Source (APS). These magnets are connected in series and are energized by only one 12-phase power supply. The eighty-first magnet is located in a temperaturecontrolled room with an NMR probe to monitor the magnetic field in the magnet and provide a reference signal for correction of the field drift due to aging of the components. The current in the magnets will be held at $497 \mathrm{~A}$. The required current stability of the power supply is $\pm 30 \mathrm{ppm}$, the current reproducibility is $\pm 50 \mathrm{ppm}$, and the current ripple is $\pm 400 \mathrm{ppm}$. The voltage required to maintain such a current in the magnets is about 1700 V. Different schemes for regulating current in the magnets are studied. Pspice software is used to simulate the behavior and the design of such a power supply under different conditions. The pros and cons of each scheme will be given and the proper power and regulating scheme will be selected.

\section{INTRODUCTION}

The storage ring of the Advance Photon Source (APS) at Argonne National Laboratory (ANL) contains 81 dipole magnets. These magnets are connected in series and fed by a 12-phase accurate and stable power supply. In order to provide a stable reference for the power supply, a 17-bit digital-to-analog converter (DAC) is programmed by the control computer. The 81st magnet is placed in a temperature-controlled room with an NMR probe to monitor the magnetic field in the magnet and provide the correction signal due to the aging of the components. The correction signal is sent to the DAC to modify the reference signal. The power supply is equipped with a passive L-C-R filter to reduce the ripple content of the output current. To regulate the current in the magnet, a high precision, low drift, zero flux current transductor is used. The transductor senses the magnet current and provides the controlling signals through the regulator. Several control loops are used to regulate the current in the magnet load. Fast correction for the line transient is provided by a relatively fast response voltage loop controlled by a high gain slow response current loop. Different design topologies are studied and will be given in the following.

\section{POWER SUPPLY SPECIFICATION}

The power supply must deliver 497 A continuously to a chain of 81 dipole magnets. The output voltage at full load operation is $1662 \mathrm{~V}$ [1].

\footnotetext{
*Work supported by U.S. Department of Energy, Office of Basic Energy Sciences under Contract No. W-31-109-ENG-38.
}

\begin{tabular}{ll}
\multicolumn{1}{c}{ REGULATION $(\Delta \mathrm{I} / \mathrm{Imax})$} \\
Stability & $\pm 30 \times 10^{-6}$ \\
Reproducibility & $\pm 50 \times 10^{-6}$ \\
Current Ripple & $\pm 400 \times 10^{-6}$ \\
Tracking Error & $\pm 100 \times 10^{-6}$
\end{tabular}

\section{DESIGN AND TOPOLOGY}

In order to provide current to the magnets, several types of power supplies are used. Phase-controlled thyristor rectifiers and switched-mode power supplies (SMPS) are the most common types used to energized the magnets. In phase-controlled power supplies a low pass filter is used to somewhat filter the ripple current. The accuracy and the response of the output current depends on the number of silicon-controlled rectifiers (SCRs) used in the power bridge and the low-pass filter frequency response. Due to the imperfection and imbalance in the power transformers, the output current may contain ripple content with the line frequency. Several techniques have been suggested in the past [2] and currently being employed to regulate the output current. To compensate for the load disturbance and ripple in the output current, active filters such as transistor banks or coupled reactors are used. The use of transistor banks means an inherent reduction in the output voltage and, consequently, lower efficiency. Use of coupled reactors can reduce the output current ripple, but reduces the tracking speed of the power supply.

The SMPSs, however, are highly efficient, quick to respond, and introduce high frequency ripple in the output current that can be filtered more easily than the low frequency ripples in the phase-controlled cases. On the other hana, the switchedmode power supplies can cause a high electromagnetic interference (EMI) due to their high di/dt and dv/dt [3]. In some cases, EMI filtering may be required to avoid perturbations to other equipment next to the power supply or the power line [4].

Another technique uses the phase-controlled and switchedmode power supplies in series combination. Usually the phasecontrolled section delivers $90 \%$ to $95 \%$ of the output power and the SMPS delivers the rest. The 5\% to $10 \%$ of the output which is delivered by the SMPS is required for the regulation. Phasecontrolled power supplies have limited bandwidth depending on the number of the SCRs used in the power bridges. For instance, in a 12-pulse phase-controlled power supply the bandwidth is limited to $720 \mathrm{~Hz}$ while the switched-mode power supply can have very large bandwidth up to $500 \mathrm{kHz}$. The utilization of the SMPS allows fast and precise correction of the output current.

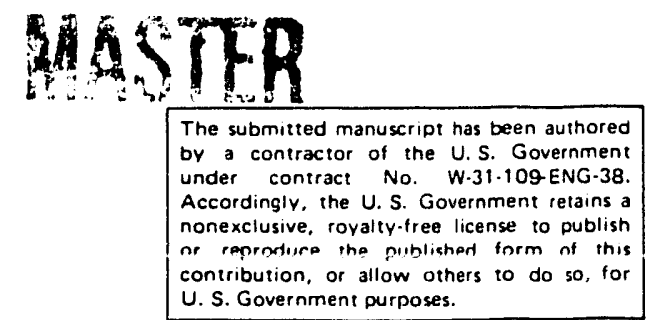




\section{DESIGN AND RESULTS}

\section{A. Filter Design}

A filter with a cut-off frequency of $720 \mathrm{~Hz}$ was designed to eliminate the fundamental and higher harmonics of the current in the power supply. The transfer function of the filter is given in the following [2]:

$$
\frac{e_{o}}{e_{i}}=\frac{s T_{2}+1}{s^{3} T_{2} L_{1} C_{1}+s^{2}\left(T_{1} T_{2}+L_{1} C_{1}+L_{1} C_{2}\right)+s\left(T_{1}+T_{2}+T_{3}\right)+1}
$$

where $T_{1}=R_{1} C_{1}, \quad T_{2}=R_{2} C_{2}, \quad T_{3}=R_{1} C_{2}, \quad L_{1}=650 \mu \mathrm{H}$, $\mathrm{C}_{1}=16200 \mu \mathrm{F}, \mathrm{C}_{2}=37800 \mu \mathrm{F}$, and $\mathrm{R}_{2}=0.265 \Omega$

However, due to imbalances in the power transformers, the $60-\mathrm{Hz}$ component passes through the filter and is noticeable in the output current if it is not filtered. The use of a pass transistor between the filter and the output can act as an active filter. The base current for such a transistor is proportional to the output current. Care must be taken to ensure a limited amount of voltage across the pass transistor. The pass transistor can fail due to high power dissipate or high voltage across it. Usually four or more control loops are used to ensure the safe and reliable operation of the pass transistor. One relatively slow but high gain current loop, a high gain output voltage loop, high gain voltage loop for $\mathrm{AC}$ line compensation, and a voltage loop to maintain certain voltage drop across the pass transistor are needed. Other loops may be used in conjunction with the above control loops. A feed forward loop is usually used for AC line voltage compensation. The limitation of this technique appears when a relatively high output voltage is required because the pass transistor must support the output voltage in the short circuit situation. This configuration is shown in Figure 1.

In order to test the above technique, a power supply with the following characteristics was prototyped.

$\begin{array}{ll}\text { Max. Output Voltage } & 80 \quad \text { V } \\ \text { Max. Output Current } & 1100 \quad \text { A } \\ \text { Stability } & \\ \text { Reproducibility } & \pm 30 \times 10^{-6} \\ \text { Current Ripple } & \pm 50 \times 10^{-6} \\ & \pm 30 \times 10^{-6}\end{array}$

A 12-phase power supply with pass transistors was designed. A set of twelve parallel Powerex wat 6 -cooled transistors (D7ST) was used for the pass transistor bank. These transistors are designed for switching applications; the voltage rating of these transistors is specified only for that purpose. In the linear application the voltage rating of the transistors must be drastically de-rated. In our case, the $400 \mathrm{~V}$ transistor was derated to $30 \mathrm{~V}$ application. Under a higher voltage application the transistor may fail. The failure under such a condition is a short circuit. In order to reduce the power drop across the transistor bank, an adaptive control loop was designed to maintain a minimum power drop across the transistor bank under different load conditions. The voltage drop across the transistor bank decreases as the load current increases, thus result in a constant power drop for all load conditions. A certain minimum voltage across the transistor bank is always needed for the current regulation. In the prototype power supply the voltage drop across the bank varied from $25 \mathrm{~V}$ to $7 \mathrm{~V}$ and load currents from $10 \mathrm{~A}$ to $1100 \mathrm{~A}$. For $\mathrm{AC}$ line voltage compensation a feed forward loop was used. Measurements were taken at full load conditions, and the ripple of $15 \mathrm{ppm}$ and stability of $45 \mathrm{ppm}$ was measured.

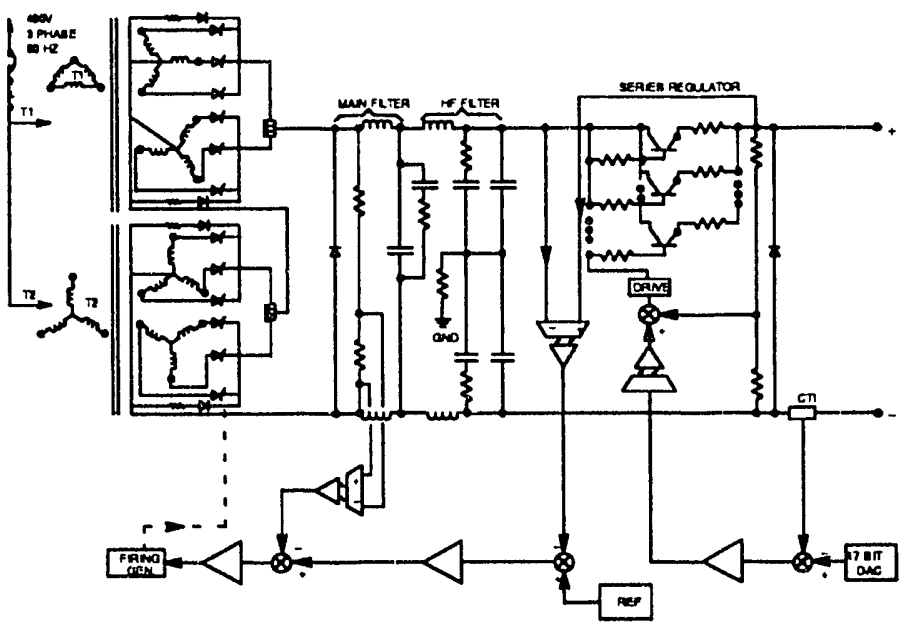

Figure 1 Block diagram for storage ring dipole power supply using phase-controlled and pass transistor.

A second alternative to the above design is the use of switched-mode power supply in conjunction with the phasecontrolled power supply. The phase-controlled part delivers the main power and the switched-mode part performs the regulation and the fine tuning of the output current. The switchedmode power supplies have a relatively larger bandwidth than the phase-controlled ones, thus the correction of the current is fast and a low ripple current can be obtained easily. For this scheme, usually about $10 \%$ of the output voltage must be delivered by the SMPS and the other $70 \%$ is delivered via the phasecontrolled power supply. The switched-mode power supply must carry the output current which is a drawback of this scheme. The instantaneous output voltage of the total power supply is ised to determine the transistor switching, while the output voltage of the SMPS is used to determine the firing angle of the thyristors. Since the output voltage of the SMPS is compared against a fixed voltage reference, the SMPS always maintains a certain portion of the output voltage. This configuration is shown in Figure 2 . The switching transistor in the SMPS must be protected against the di/dt and $\mathrm{dv} / \mathrm{dt}$ due to the output filter and the magnetic load. The SMPS in Figure 2 is fed from bridge rectifier with a typical R-L-C filter. The filter characteristic may cause oscillation in conjunction with the SMPS and its load. The filter is usually designed to be near critically damped to improve the overall efficiency. The R-L-C filter can give rise to low frequency oscillations at its output when it is subject to periodic load changes, e.g. when supplying power to an SMPS. In order to avoid such a problem, the inductance in the R-L-C filter must be reduced and the R and C increased [5]. 


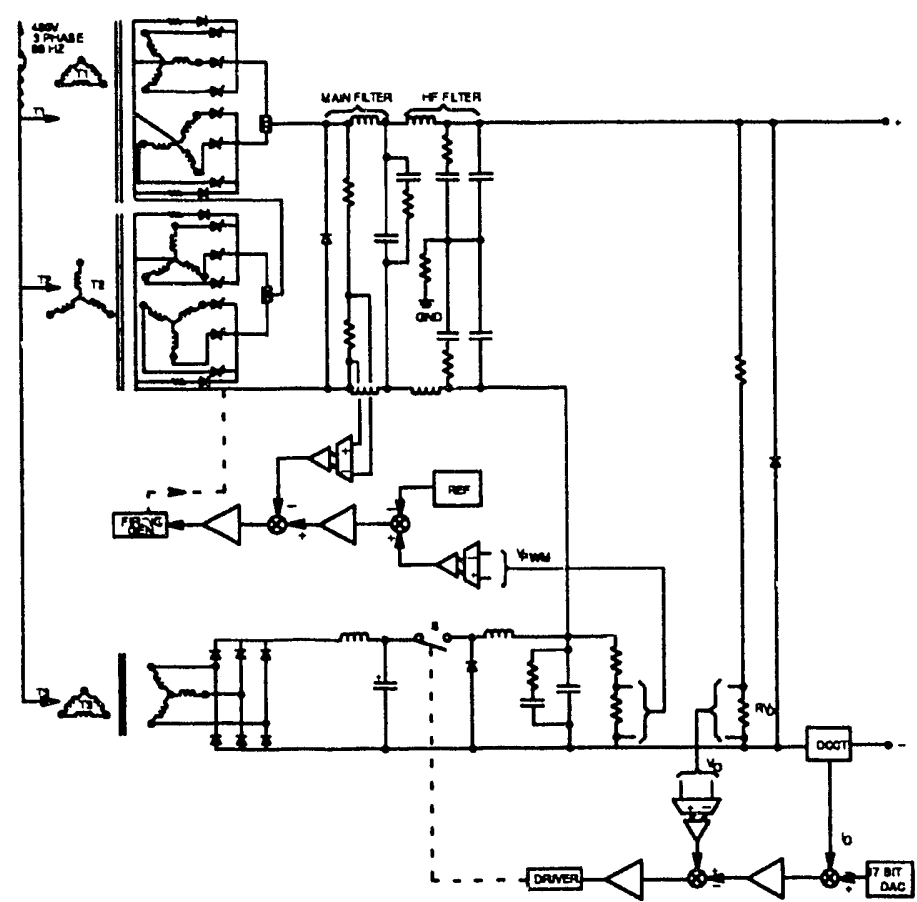

Figure 2 Block diagram for storage ring power supply using phase-controlled and switched-mode power supply

\section{CONCLUSION}

Different configurations for the power supply of the storage ring dipole magnets were studied. In order to eliminate the output current ripple, an active filter was needed. Use of a pass transistor as the active filter was investigated and it was concluded that complicated control circuitries are needed to make such a scheme work. It was also concluded that under high output voltage, the pass transistor may fail. A prototype was built to investigate the operation of such a configuration, and the results indicate almost one order magnitude better performance than the required regulation.

The use of SMPS in conjunction with phase-controlled power supply was also investigated and the required control loops were shown. The SMPS may cause oscillation when the i. nut filter inductance is relatively high. The switching transistor must be protected against di/dt and dv/dt due to the output filter and load inciuctances.

\section{REFERENCES}

[1] Advanced Photon Source Design Handbook Vol. II, Dec. 1989.

[2] W. F. Praeg, "A high-current low pass filter for magnet power supplies," IEEE Trans. Industrial Electronics and Control Instrumentation, Vol. IECI-17, No.1, pp. 16-22, Feb. 1970.

[3] N. Mohan, T. M. Underland, and W. P. Robbinson, Power Electronics Converters. Application and Design, (New York: John Wiley \& Sons, Inc. 1987).
[4] J. A. Pomilo, D.Wisnivesky, and A. C. Lira, "A Novel Topology for the Bending Magnets Power Supply at LNLS," IEEE Trans. on Nuclear Science, Vol. 39, No. 5, pp. 1506-1511, Oct. 1992.

[5] M. Fathizadeh, O. D. Despe, D. G. McGhee, "Analysis and Correction of Noise on Elecironic Circuits in an Accelerator Environment," Nuclear Science Symposium and Medical Imaging Conference, Orlando, Florida, 1992.

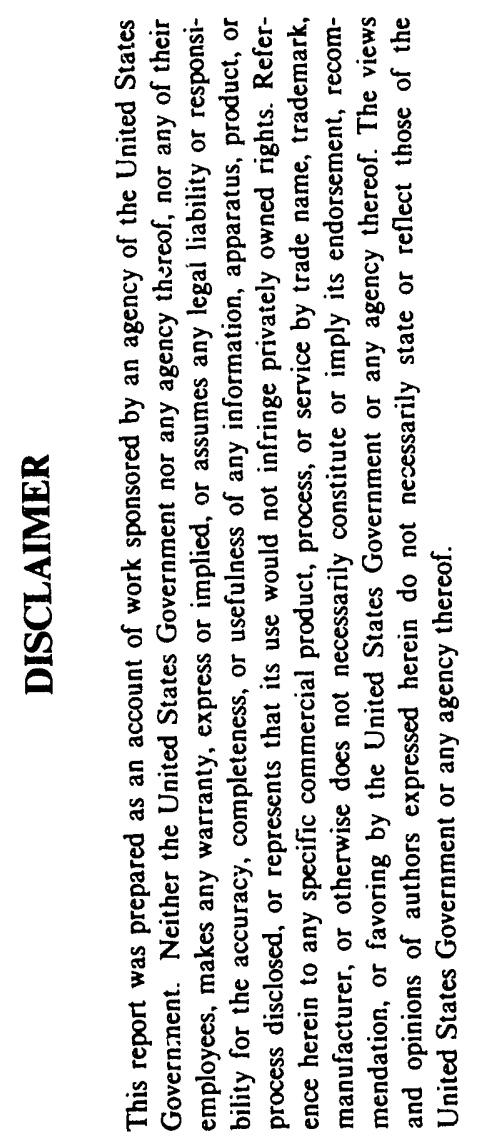



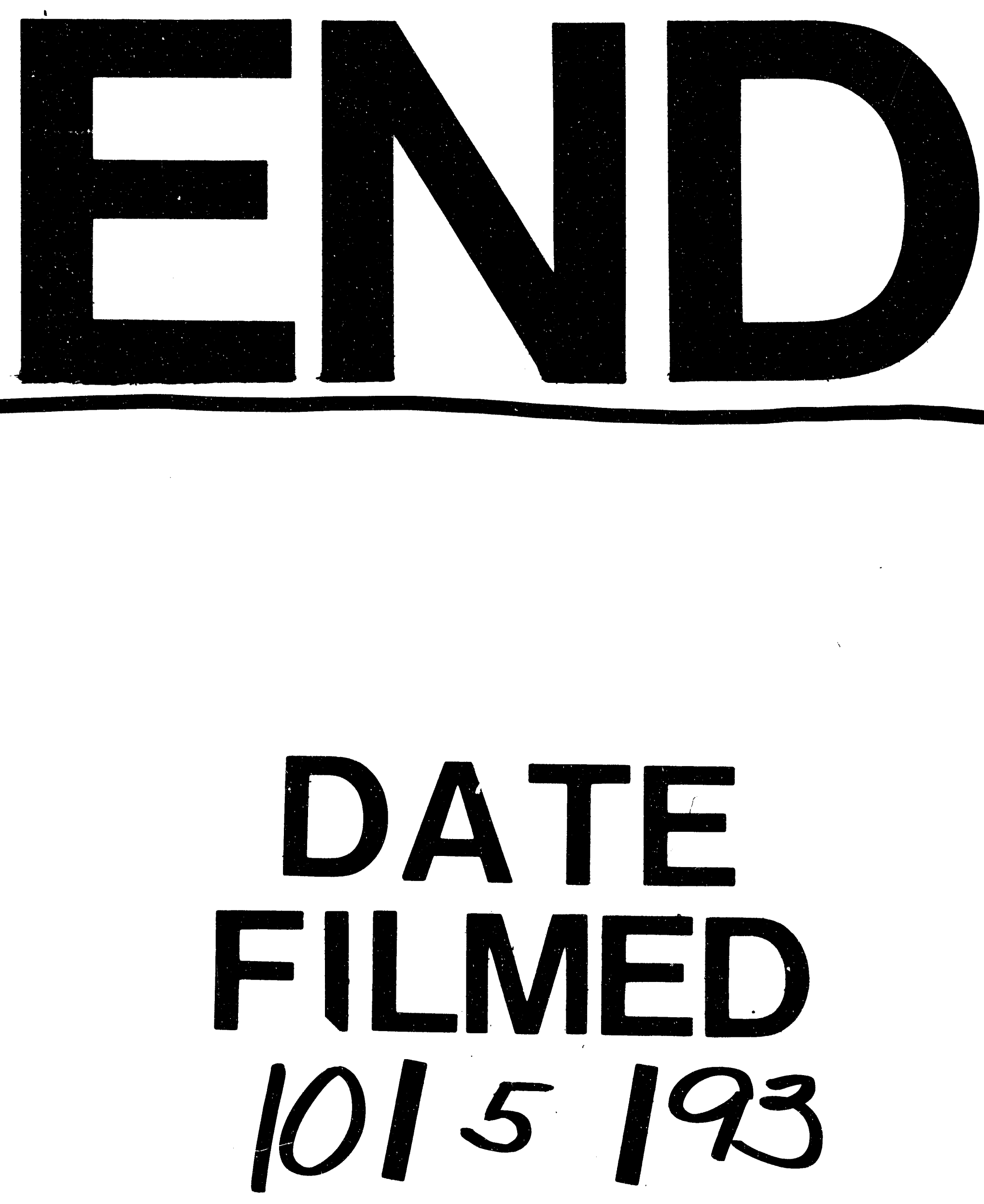

I 


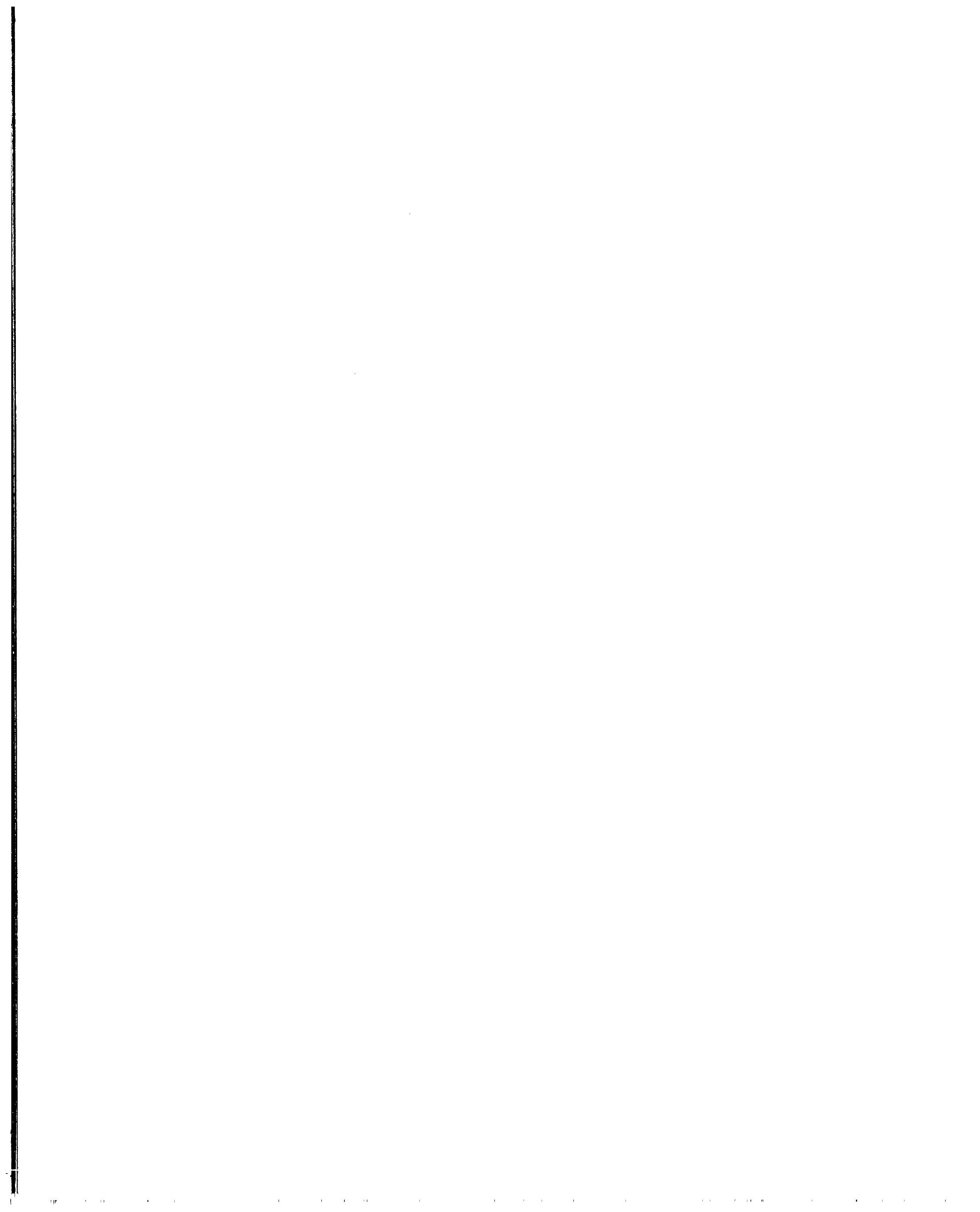

